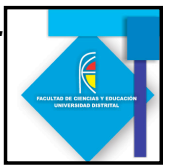

\title{
EXPERIENCIAS DE AULA CON MATERIALES MAGNÉTICOS Y CARACTERIZACIÓN DEL MAGNETISMO EN LA ESCUELA SECUNDARIA
}

\section{CLASSROOM EXPERIENCES WITH MAGNETIC MATERIALS AND MAGNETIC CHARACTERIZATION OF HIGH SCHOOL}

\author{
Oscar Moreno Fernández ${ }^{1}$ \\ Adonai Basto Rueda²
}

\begin{abstract}
Resumen
El siguiente trabajo, tiene como propósito enriquecer la experiencia y la relación estudiante-fenómeno magnético, para la obtención de postulados respecto a las teorías sobre magnetismo, reconociendo en el aula de clase, cómo los estudiantes de secundaria de grados sexto, decimo y undécimo del Liceo Normandía, pueden caracterizar el fenómeno del magnetismo. Esto, a partir de la interacción y observación de determinados tipos de materiales magnéticos, como ferromagnéticos y paramagnéticos. Lo que se pretende, es desde un trabajo experimental, mostrar la forma en que los estudiantes interpretan los fenómenos magnéticos con experiencias sencillas, obteniendo análisis detallados sobre las formas de explicarlos.
\end{abstract}

Palabras clave: Magnetismo, Hipótesis, Experiencias y Fenómeno.

\begin{abstract}
The following article is proposed to improve the experience and the relation studentphenomenon, to obtain assumptions for theories of magnetism, recognizing how high school students in grades sixth, tenth and eleven of the school Liceo Normandia can characterize the phenomenon of magnetism. This is based on the interaction and observation of certain types of magnetic materials, such as, ferromagnetic and paramagnetic among others. The requirement is from an experimental study, showing the way in the students interpret magnetic phenomenon with simple experiments, obtaining a detailed analysis about ways to explain it.
\end{abstract}

Keywords: magnetism, hypothesis, scenario and Phenomenon.

\footnotetext{
${ }^{1}$ Universidad Pedagógica Nacional,

gauus_bela@hotmail.com

${ }^{2}$ Universidad Pedagógica Nacional, oscarmorenoupn@yahoo.com
} 


\section{Introducción}

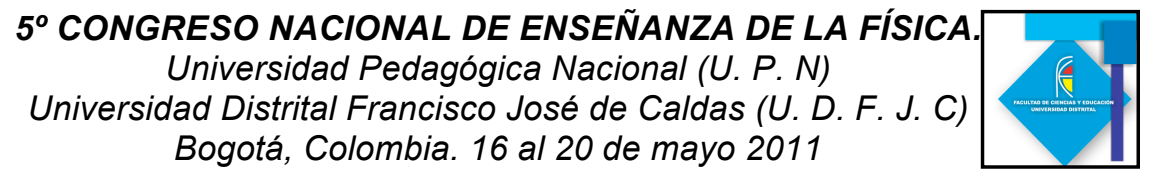

No sabe con certeza en qué momento se reflexiono sobre el fenómeno magnético, pero si se puede hacer una descripción histórica y cultural de la evolución del fenómeno, está a su vez es nuestro punto de partida para plantear algunas didácticas en el aula las cuales se dividieron de acuerdo con estas descripciones. Se tienen algunos indicios de que los griegos fueron los primeros en reflexionar sobre el magnetismo, las diferentes leyendas nos conducen a pensar que fue la interacción con las piedras magnéticas lo que conllevó a diferentes tipos de reflexiones, siguiendo esta línea la piedra magnética es utilizada como un instrumento de orientación, tiempo después tenemos a William Gilbert de Colchester con su método experimental el cual no deja nada a la especulación, y se concibe como el fundador de la ciencia del magnetismo. (Martina y Tagüeña, 1997) En el orden histórico antes mencionado se pretenden desarrollar experiencias de aula que validen el conocimiento propio del estudiante el cual me permite desarrollar la evolución del fenómeno magnético, y de esta forma construir su aprendizaje; el maestro debe constantemente diseñar estrategias que le permitan evidenciar las formas en que los estudiantes comprenden los fenómenos, esto es concebir la participación para poder estructurar su aprendizaje. (Moreira, M. y Greca, I., 2004) Se plantean 5 momentos de la experiencia de aula que serán ampliados más adelante

1. test sobre el magnetismo

2. Interacción con materiales magnéticos

3. Construcción de una brújula

4. Algunas nociones de campo magnético

\section{Test sobre el Magnetismo}

Este test se desarrolló para obtener información sobre la interpretación de algunos conceptos como: Mono polos de un imán, comportamiento magnético, atracción y repulsión; se formularon preguntas de opción múltiple con única respuesta usando algunos distractores, fueron ilustradas y se representaron los polos magnéticos con colores para no mencionar los conceptos, luego del desarrollo de la actividad obtuvimos información suficiente, la cual nos sirvió para estructurar preguntas mejor elaboradas.

\section{Interacción con Materiales Magnéticos}

Se llevan a las aulas materiales magnéticos de diferentes formas y tamaños para que los estudiantes interactúen, se cuestionen sobre lo que observan y experimentan, conformando grupos de trabajo lo cual posibilita el debate de los diferentes tipos de reflexiones. Algunos de los imanes se cubrieron con silicona para diferenciarlos de los demás, esto con el fin de que fueran reconocidos por sus propiedades magnéticas y no por su forma. Los estudiantes interactuaron con todo tipo de materiales y empezaron a deducir algunas propiedades ya conocidas en la antigüedad como la repulsión y la atracción magnética estos son algunos ejemplos de sus observaciones e interpretaciones del fenómeno magnético:

"Pudimos observar que el imán pequeño se pegaba al grande con una fuerza que los atraía: materiales taza de plástico, llaves, mano. La aguja, pudimos observar que el magnetismo y la 


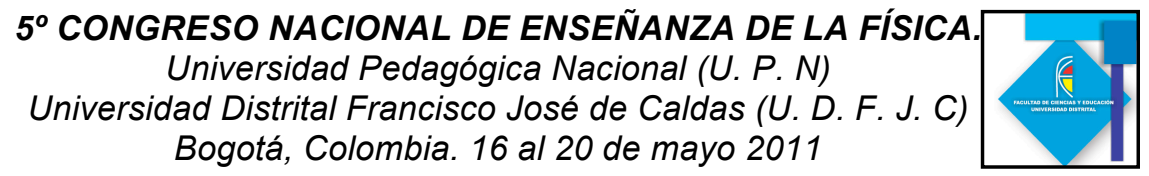

fuerza las atrae por que los dos son positivos, material imán y aguja. El corcho tiene una aguja incrustada por el medio cuando se pasa el imán grande se atraen. Estas son algunas de las observaciones realizadas por estudiantes de grado sexto"

"cuando está en + se voltea a -, si hacemos girar la aguja sale volando y si la ponemos la aguja acostada se pega al imán y no la suelta, uno coge una mandarina le pone un imán encima de la mandarina y uno debajo y el imán se mueve"

"pues yo creo que como que tiene ondas que atrae al metal como lo de un agujero negro pero solo con el metal"

\section{Construcción de la Brújula}

En la construcción de la brújula se abarcaron varios aspectos del magnetismo como imantar una aguja, y la orientación que muestra está en diferentes campos (magnético y electromagnético). En la figura 1 se puede observar el dispositivo construido por los estudiantes.

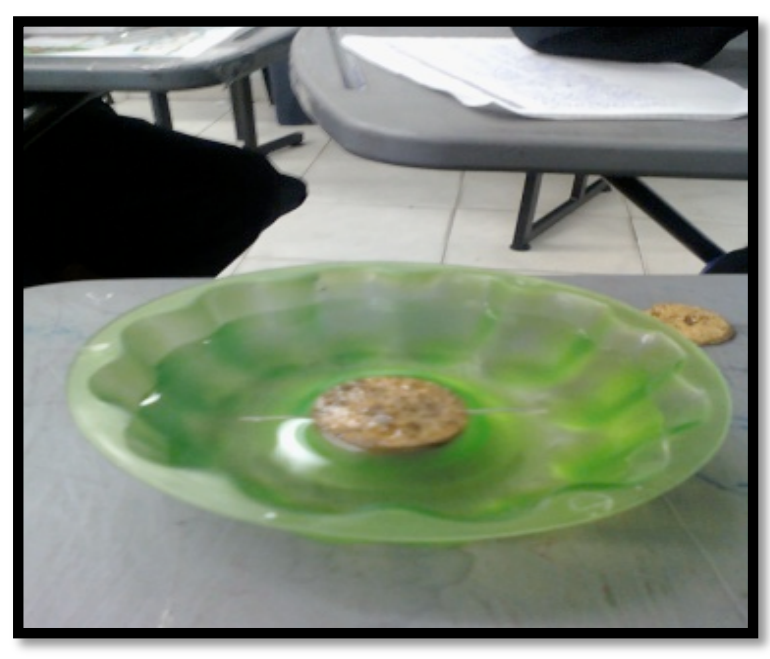

Figura 1

\section{Algunas Nociones de Campo Magnético}

Inicialmente se les pidió a los estudiantes que representaran por medio de un dibujo los movimientos de los diferentes materiales y lo que causaba el movimiento. En la figura numero 2 se muestra el dibujo de un estudiante de sexto el cual intenta dar una explicación al fenómeno del movimiento sin fuerzas de contacto.

Del cuerpo metálico del diseño se puede observar que cuando el imán esta cerca sale de los objetos un tipo de líneas discretas, las cuales nunca aparecen en el objeto de plástico, esto conllevaría a que el imán solo afecta a los elementos metálicos. 


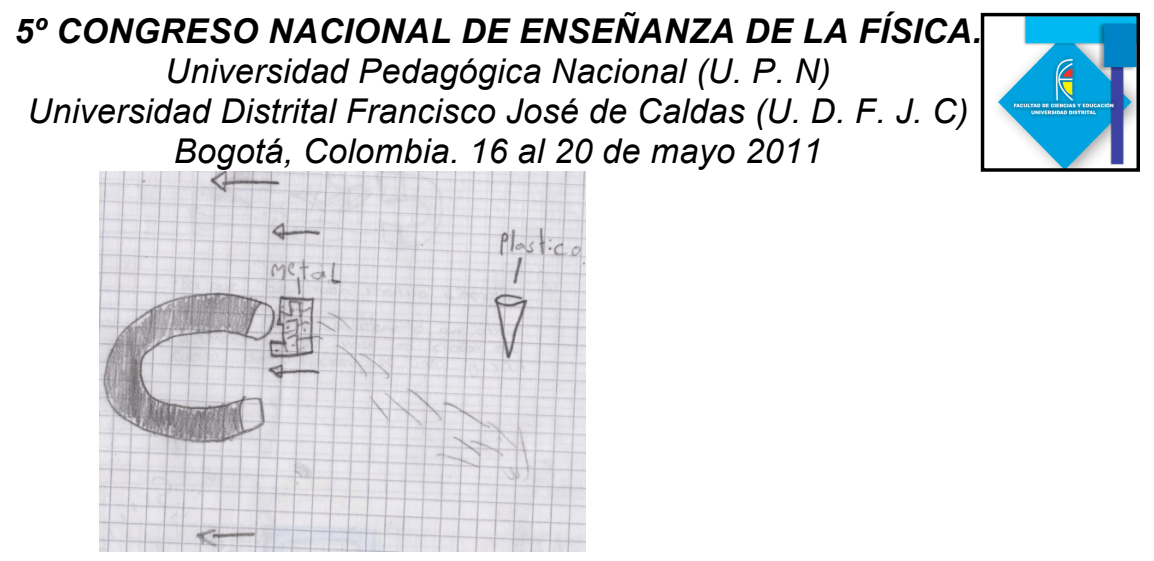

Figura 2

En la figura número 3 que es realizada por un estudiante de grado decimo se observan líneas continuas en un solo plano que también solo afectan los clavos siendo estos metálicos, al estar cerca estas líneas del objeto producen una ondulación como respuesta a su proximidad.

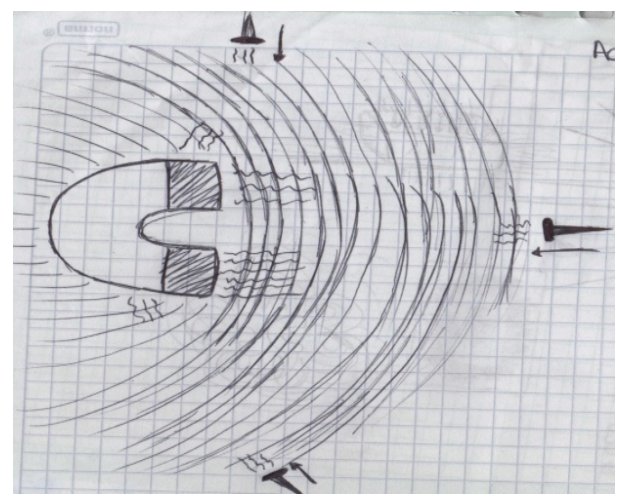

Figura 3

Por último la figura numero 4, nos muestra limaduras de hierro en presencia del campo magnético, experiencia que se realizo después de tener las representaciones de cada uno de los estudiantes.

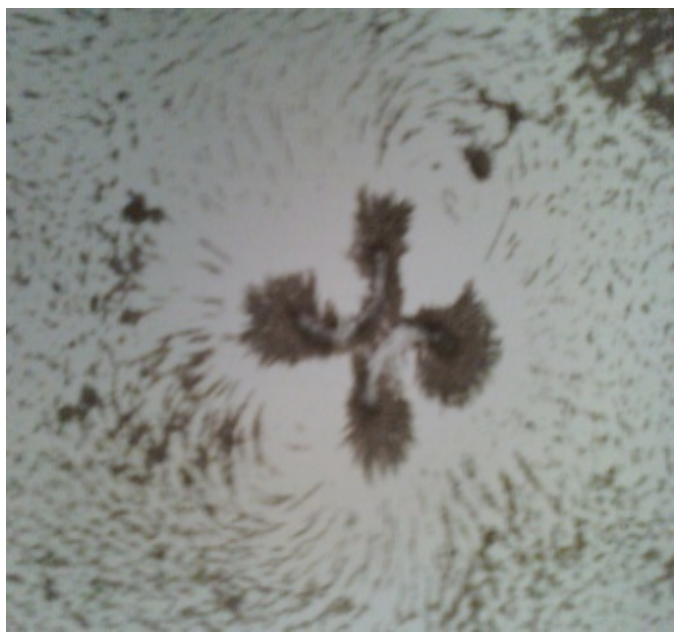

Figura 4 


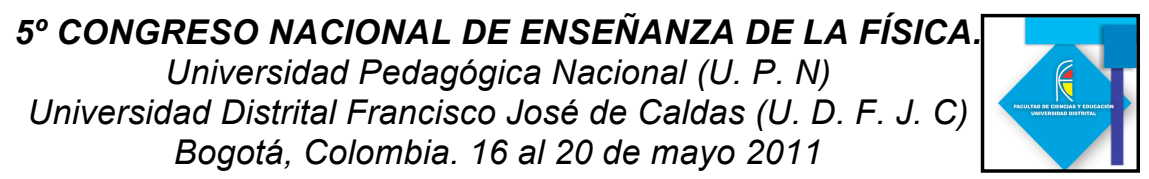

\section{Conclusiones}

Al incluir estas experiencias que se pueden transportar fácilmente al aula, se consigue revelar a los estudiantes fenómenos que muestran principios básicos para el inicio del estudio del magnetismo, motivándolos positivamente, gracias a la observación e interacción con el fenómeno magnético, gracias a esto se obtuvo toda una serie de razonamientos que nos permiten direccionar de una manera más adecuada este tipo de fenómeno.

\section{Referencias bibliográficas}

Martina, E. y Tagüeña, J (1997) De la brújula al espín. México DF: Fondo de cultura económica, S.A. DE C. V.

Moreira, M. y Greca, I., 2004). Obstáculos representacionales mentales en el aprendizaje de Conceptos cuánticos, en Cambio conceptual, obstáculos representacionales, modelos mentales, esquemas de asimilación y campos conceptuales (UFRGS, Porto alegre)

Gilbert, W. (1600). Magnete Magnetiasque Corporibus et de Magno Magnete Tellure Physiologia Nova. Londres: Dover Publications, Inc.

Rudolf F. G. (1968).traducido por Postigo L. Electricidad experimental para todos. Barcelona. Editorial Ramón Sopena, S.A. 